\title{
Is There a Role for Radiopaque Markers in Identifying Defecation Disorders?
}

\author{
Yoo Jin Lee \\ Division of Gastroenterology and Hepatology, Department of Internal Medicine, Keimyung University School of Medicine, Daegu, Korea
}

\author{
Article: Rectosigmoid localization of radiopaque markers for identifying defecation disorders in patients with chronic \\ constipation: a retrospective cohort study \\ Abe T, Kunimoto M, Hachiro Y, Ohara K, Inagaki M, Murakami M \\ (J Neurogastroenterol Motil 2021;27:419-425)
}

Constipation is a highly prevalent condition which has negative impact on a patient's quality of life. ${ }^{1,2}$ Functional constipation (FC) is categorized into 3 subtypes according to their pathophysiologic mechanism: normal transit constipation (NTC), slow transit constipation (STC), and defecatory disorders (DD). ${ }^{3}$ Classification of FC subtypes provides valuable insights to the underlying pathophysiology of FC. However, patients with FC complain of similar but diverse symptoms, and each subtype often overlaps, making it difficult to classify patients with FC based on symptoms. As a result, clinicians have difficulties choosing an appropriate treatment, and patients are often dissatisfied with the therapy prescribed. ${ }^{2}$

DD is characterized by difficulty in rectal evacuation from inappropriate rectal propulsive forces and/or paradoxical contraction of the pelvic floor and external anal sphincter during attempted defecation. ${ }^{4}$ Patients with DD do not benefit from fiber or laxatives, and their symptoms may worsen in some cases. ${ }^{5}$ In particular, patients with DD respond less to laxatives and respond best to biofeedback therapy. ${ }^{3}$ Biofeedback therapy showed a favorable outcome even in cases of DD coexisting with STC or NTC, suggesting a potential influence of outlet dysfunction on a delayed colonic transit. ${ }^{6,7}$ Thus, early subtyping of FC is crucial for selecting suitable candidates for biofeedback therapy regardless of STC overlap. According to the Rome IV criteria, DD can be demonstrated by 2 of 4 abnormal findings on objective anorectal tests such as manometry or anal surface electromyography, balloon expulsion test, or barium or magnetic resonance defecography. ${ }^{8}$ However, anorectal tests are not universally available in real clinical practice.

Radiopaque markers are non-invasive, easily accessible, and inexpensive modality for accessing colonic transit time (CTT). ${ }^{4}$ Despite of the convenience of radiopaque markers, its diagnostic feasibility for DD seems to be somewhat disappointing in the existing literature. Several studies have reported that rectosigmoid (RS) accumulation of radiopaque markers was not useful to diagnose DD or discriminate DD from NTC and/or STC.$^{9-11}$ However, Nullens et al $^{12}$ suggested that regional scintigraphic transit profiles can differentiate DD from STC, suggesting diagnostic value of non-invasive measurement of colonic transit.

In this regards, Abe et $\mathrm{al}^{13}$ evaluated the diagnostic value of RS localization of radiopaque markers in discriminating constipation subtypes. Among 169 patients with refractory constipation, 79 (46.7\%) patients were confirmed to have DD based on standard anorectal tests. Notably, the RS accumulation was useful for dis-

Received: May 31, 2021 Revised: None Accepted: June 10, 2021

() This is an Open Access article distributed under the terms of the Creative Commons Attribution Non-Commercial License (http://creativecommons. org/licenses/by-nc/4.0) which permits unrestricted non-commercial use, distribution, and reproduction in any medium, provided the original work is properly cited.

${ }^{*}$ Correspondence: Yoo Jin Lee, MD Division of Gastroenterology and Hepatology, Department of Internal Medicine, Keimyung University School of Medicine, 1035 Dalgubeol-daero, Dalseo-gu, Daegu 42601, Korea

Tel: +82-53-258-7739, Fax: +82-53-258-4349, E-mail: doctorlyj@gmail.com 
criminating DD from STC with a sensitivity of $62.5 \%$, specificity of $88.2 \%$, and a positive likelihood ratio of 5.3. In discriminating between DD and NTC, the specificity of RS accumulation was $82.1 \%$, however, the sensitivity was only $10.6 \%$.

Interestingly, RS accumulation was not found in $70.9 \%$ of NTC cases, those lead to extremely low sensitivity (10.6\%) and failed discrimination between DD and NTC in this study. Similarly, radiopaque markers cannot reach to the RS location in an abundant numbers of patients in DD with STC, which resulted in insufficient sensitivity (62.5\%). These findings are thought to have originated from fundamental drawback of CTT using radiopaque markers. In order to more accurately categorize the subtypes of $\mathrm{FC}$, various anorectal tests are needed as recommended on the guidelines. ${ }^{3,8}$

The current study is limited by the fact that a single capsule ingestion followed by single abdominal film technique was applied. This method may underestimate delayed CTT. ${ }^{14}$ However, it is necessary to interpret the findings of this study with a focus on the fact that CTT using radiopaque markers can be easily performed even in primary care settings where other anorectal tests are not possible. Although such a design may limit generalization of the current findings, a single capsule ingestion followed by single abdominal film technique seems to have sufficient value as a primary test for patients with $\mathrm{FC}$ in terms of being able to discriminate $\mathrm{DD}$ from STC with a simple method.

The authors also suggested the diagnostic algorithm for predicting patients with DD. Since CTT using radiopaque markers is an inexpensive and convenient method, the algorithm proposed by the authors can be applied as an initial diagnosis of $\mathrm{FC}$, especially in the absence of specialized anorectal test for assessing FC. With this approach, the primary care clinicians may be able to identify patients for referral to a specialist, such as patients who require further anorectal tests or those who will benefit from biofeedback therapy. However, when using CTT with radiopaque markers in clinical practice, it should be borne in mind that there exists significant heterogeneity in the method of measuring CTT among studies, and this may have influenced the study results. ${ }^{15}$ Thus, further validations of the proposed protocol in this study is required before clinical application.

Taken together, RS accumulation of radiopaque markers seems to be helpful for discriminating DD from STC when anorectal testing are not available. However, RS accumulation of radiopaque markers may be insufficient to exclude DD because it does not adequately discriminate DD from NTC. The proposed diagnostic approach for constipated patients in this study is simple and practical, but requires further validation.
Financial support: None.

\section{Conflicts of interest: None.}

\section{References}

1. Suares NC, Ford AC. Prevalence of, and risk factors for, chronic idiopathic constipation in the community: systematic review and metaanalysis. Am J Gastroenterol 2011;106:1582-1591.

2. Johanson JF, Kralstein J. Chronic constipation: a survey of the patient perspective. Aliment Pharmacol Ther 2007;25:599-608.

3. Shin JE, Jung HK, Lee TH, et al. Guidelines for the diagnosis and treatment of chronic functional constipation in Korea, 2015 revised edition. J Neurogastroenterol Motil 2016;22:383-411.

4. Bharucha AE, Lacy BE. Mechanisms, evaluation, and management of chronic constipation. Gastroenterology 2020;158:1232-1249, e3.

5. Eswaran S, Muir J, Chey WD. Fiber and functional gastrointestinal disorders. Am J Gastroenterol 2013;108:718-727.

6. Chiotakakou-Faliakou E, Kamm MA, Roy AJ, Storrie JB, Turner IC. Biofeedback provides long-term benefit for patients with intractable, slow and normal transit constipation. Gut 1998;42:517-521.

7. Rao SS, Patcharatrakul T. Diagnosis and treatment of dyssynergic defecation. J Neurogastroenterol Motil 2016;22:423-435.

8. Mearin F, Lacy BE, Chang L, et al. Bowel disorders. Gastroenterology Published Online First: 18 Feb 2016. doi: 10.1053/j.gastro.2016.02.031.

9. Grotz RL, Pemberton JH, Talley NJ, Rath DM, Zinsmeister AR. Discriminant value of psychological distress, symptom profiles, and segmental colonic dysfunction in outpatients with severe idiopathic constipation. Gut 1994;35:798-802.

10. Staller K, Barshop K, Ananthakrishnan AN, Kuo B. Rectosigmoid localization of radiopaque markers does not correlate with prolonged balloon expulsion in chronic constipation: results from a multicenter cohort. Am J Gastroenterol 2015;110:1049-1055.

11. Cowlam S, Khan U, Mackie A, Varma JS, Yiannankou Y. Validity of segmental transit studies used in routine clinical practice, to characterize defaecatory disorder in patients with functional constipation. Colorectal Dis 2008;10:818-822.

12. Nullens S, Nelsen T, Camilleri M, et al. Regional colon transit in patients with dys-synergic defaecation or slow transit in patients with constipation. Gut 2012;61:1132-1139.

13. Abe T, Kunimoto M, Hachiro Y, Ohara K, Inagaki M, Murakami M. Rectosigmoid localization of radiopaque markers for identifying defecation disorders in patients with chronic constipation: a retrospective cohort study. J Neurogastroenterol Motil 2021;27:419-425.

14. Bouchoucha M, Thomas SR. Error analysis of classic colonic transit time estimates. Am J Physiol Gastrointest Liver Physiol 2000;279:G520G527.

15. Miller LE, Ibarra A, Ouwehand AC. Normative values for colonic transit time and patient assessment of constipation in adults with functional constipation: systematic review with meta-analysis. Clin Med Insights Gastroenterol Published Online First: 31 Aug 2017. doi: $10.1177 / 1179552217729343$. 